SSC-250/Rev.

\title{
REPORT OF THE AD HOC COMMITTEE ON SSC PHYSICS
}

\author{
Superconducting Super Collider Laboratory* \\ 2550 Beckleymeade Avenue \\ Dallas, TX 75376
}

December 11, 1989

Revised: April 1990

* Operated by Universities Research Association, Inc., for the U.S. Department of Energy under Contract No. DE-AC02-89ER40486. 


\section{DISCLAIMER}

This report was prepared as an account of work sponsored by an agency of the United States Government. Neither the United States Government nor any agency thereof, nor any of their employees, makes any warranty, express or implied, or assumes any legal liability or responsibility for the accuracy, completeness, or usefulness of any information, apparatus, product, or process disclosed, or represents that its use would not infringe privately owned rights. Reference herein to any specific commercial product, process, or service by trade name, trademark, manufacturer, or otherwise does not necessarily constitute or imply its endorsement, recommendation, or favoring by the United States Government or any agency thereof. The views and opinions of authors expressed herein do not necessarily state or reflect those of the United States Government or any agency thereof. 


\section{DISCLAIMER}

Portions of this document may be illegible in electronic image products. Images are produced from the best available original document. 
SSC-250/Rev.

\author{
REPORT OF THE AD HOC \\ COMMITTEE ON SSC PHYSICS \\ Superconducting Super Collider Laboratory \\ 2550 Beckleymeade Avenue \\ Dallas, TX 75376
}

December 11, 1989

Revised: April 1990

\title{
1. INTRODUCTION
}

An Ad Hoc Committee on SSC Physics was convened by the Director of the SSC Laboratory with the general charge of evaluating the impact of variations in the SSC energy and luminosity on the physics program. The details of this charge are given in Appendix I. The Committee, whose members are listed in Appendix II, met at the SSC Laboratory on November 30 and December 1, 1989.

The proposed SSC, as documented in the Conceptual Design Report SSC-SR$2020,[1]$ consists of proton-proton storage rings with a maximum center-of-mass (c.m.) energy of $40 \mathrm{TeV}$ and a design luminosity of $10^{33} \mathrm{~cm}^{-2} \mathrm{sec}^{-1}$. These energy and luminosity specifications are the result of many previous studies that examined physics goals as well as capabilities of accelerator and detector technologies. One of the central physics themes of the SSC is the investigation of electroweak symmetry breaking, a phenomenon that is expected to produce observable, new 
effects at an energy scale at or below the TeV level. The detection of these effects requires beam energies that are at least an order of magnitude higher since the constituent particles within a proton, which initiate the basic interactions, typically carry only a small fraction of the proton energy.

The design luminosity of $10^{33} \mathrm{~cm}^{-2} \mathrm{sec}^{-1}$ represents a value that can be achieved with confidence using presently known accelerator techniques. The ultimate peak luminosity of the SSC, as limited by beam-beam perturbations and synchrotron power dissipation, may exceed the $10^{34} \mathrm{~cm}^{-2} \mathrm{sec}^{-1}$ level. However, this level is unlikely to be reached during the initial years of SSC operation. Moreover, such gains in peak luminosity do not translate directly into equivalent gains in the average luminosity because of the resulting decrease in beam lifetimes. [2] Finally, the detection and measurement of proton-proton interactions are already very challenging at the design luminosity and become more difficult as the luminosity is raised significantly beyond this level.

The construction cost of the SSC depends rather directly on its maximum energy since the physical size of the SSC scales with energy. Reductions in beam energy will result in a decrease of the cross sections of nearly all physical processes of interest. Since event rates are given by the product of cross section and luminosity, such decreases can, in principle, be compensated by operating the SSC at higher luminosities. However, the operation of the accelerator and detectors will place a limit on achievable or usable luminosities. The SSC maximum energy is, therefore, the basic parameter that determines the ultimate physics reach of the machine. 
The Ad Hoc Committee on SSC Physics has reexamined the relationship between beam energy, machine luminosity, and physics capability. In the next section, the physics motivation for the SSC is reviewed in general terms. This is followed by a discussion of the ability to detect a number of specific processes as a function of the SSC energy and luminosity. The viability of various detector technologies is then assessed as a function of luminosity. The report ends with a brief summary and some conclusions.

\section{PHYSICS GOALS OF THE SSC}

The Superconducting Super Collider (SSC) is designed to help us answer the most fundamental questions about nature: what are things made of and what are the forces between those constituents? In the last fifteen years, enormous advances have been made in our understanding of these questions. We have learned that ordinary matter consists of electrons, which are leptons, and quarks, which combine to make the protons and neutrons found in the nucleus. We know that the forces acting on these particles are carried by the photon (the particle of light), $W$ bosons (responsible for certain forms of radioactivity), the $Z$ boson (related to the $W$ boson), and gluons (associated with the strong force that binds the quarks into objects such as the proton, neutron, and pion).

The quarks and leptons can be arranged into three families which display great similarities in their behavior. One of the quarks, the $t$, has yet to be observed directly. Today we have no understanding of why these families exist or why there seem to be just three of them. We also do not know whether there is another level of structure beneath the quarks and leptons. 
To answer such questions and to learn whether there are additional forces beyond those carried by the photon, W, Z, and gluons, we need to probe deep inside matter. Just as Rutherford inferred the structure of the atom by bombarding it with alpha particles in 1909, we seek the very highest energy beams to penetrate to the innermost parts of matter. The present state of knowledge has been obtained with machines reaching total energies of nearly $2 \mathrm{TeV}$ (trillion electron volts). To find answers to our present questions calls for a dramatic increase in that energy.

The exploration of new phenomena requires great energy because the objects we seek are massive. Such objects are produced only rarely and, therefore, it is essential that the beams have high intensity (luminosity), as well as high energy. The energy and luminosity of the machine are the fundamental parameters that determine what can be produced and at what rate. In addition to the machine that creates new particles, we also require enormous detectors for their observation. These detectors must be able to find the few, rare events of great interest that are hidden in a data stream generated by 100 million interactions per second.

We can delineate goals for the SSC in general terms. This has been done numerous times and the main topics can be stated in the form of questions: Are there new forces? What is the source of mass? Are there constituents inside quarks and leptons? These questions may well be answered by the existence of new particles, unobservable in lower energy machines. The primary goals for the SSC include searches for new $W$ and $Z$ bosons, Higgs bosons, technicolored or supersymmetric particles, and indications of quark and lepton substructure. 
We do not know precisely the energy scale of the new physics. The higher the energy of the machine and the more intense its beams, the better the chance we have of discovering something new and fundamental. The SSC Conceptual Design Report calls for a machine with $20 \mathrm{TeV}$ beams giving a total energy of $40 \mathrm{TeV}$. The luminosity was specified to be $10^{33} \mathrm{~cm}^{-2} \mathrm{sec}^{-1}$. The SSC would provide a factor of more than twenty in energy over the biggest existing machine and a factor of several hundred in luminosity. This report evaluates the implications for the SSC physics program of varying the energy in the range from 30 to $40 \mathrm{TeV}$. We also consider how the capability of the SSC to operate eventually at luminosities above $10^{33} \mathrm{~cm}^{-2} \mathrm{sec}^{-1}$ would enhance the ultimate potential for discoveries.

The goal of the SSC experiments is the discovery of new phenomena. Some phenomena would be unmistakable. A new, massive $Z$-boson provides a good example. Almost nothing can produce the same pattern in a detector as a new $Z$ and the observation of a few events would be convincing. On the other hand, some new phenomena may be obscured by background processes and it is necessary then to produce hundreds or thousands of events. It is generally advantageous to run at high energy since the probability (cross section) for producing the events of interest increases with energy. While a given signal event rate can also be achieved at a somewhat lower energy but higher luminosity, signal-to-background ratios typically become smaller with decreasing energy and can prevent a tradeoff between energy and luminosity. Moreover, machine and detector capabilities will place a limit on the maximum luminosity at which the SSC can be operated. 
Our present understanding of particle physics is summarized in the Standard Model, which enables us to calculate the forces between the known quarks and leptons. However, it provides no explanation of the masses of these particles or why the particles come in the patterns they do. In the Standard Model, all particles initially lack mass and acquire it only through a process called "electroweak symmetry breaking." How this actually occurs is unknown. The simplest model requires the existence of a single new particle called the Higgs boson. Other more complicated models postulate new classes of particles such as technicolored or supersymmetric particles or additional Higgs bosons. A major goal of the SSC is to discover the true nature of electroweak symmetry breaking by finding evidence for any of these hypothetical particles. Since the masses of such particles are not known, the search must be conducted over as wide a range as possible. We do have good reason to believe that the clues to electroweak symmetry breaking lie at or below the $1 \mathrm{TeV}$ scale. For each choice of machine energy and luminosity there is a domain of sensitivity to Higgs and other new particles. As shown below, increasing energy and luminosity expand these domains. For the variations considered in this report, the SSC will expand the explorable mass ranges by more than a factor of ten.

The SSC could provide direct evidence for new forces in nature. Just as the hadron collider at CERN disclosed the existence of $\mathrm{W}$ and $Z$ bosons, the SSC could find similar particles with masses up to several (perhaps ten) $\mathrm{TeV}$, an increase of more than an order of magnitude beyond the capability of today's highest energy machine, the Fermilab Tevatron. Exactly how far this explo- 
ration could proceed will depend on the energy and luminosity of the machine, as detailed below, but the potential for discovery is extraordinary.

At every stage in our exploration of matter, what appeared to be fundamental constituents turned out to be divisible still further. Atoms were found to contain electrons and nuclei. The nucleus was discovered to consist of protons and neutrons. Now we know that quarks are the constituents of the protons and neutrons. The search for particles within the quarks can be parametrized by a decreasing size, or conversely, by an increasing energy scale. Today we can set limits on the scale of quark or lepton compositeness close to the $\mathrm{TeV}$ level. The SSC would allow explorations up to scales of $30 \mathrm{TeV}$. The precise domain explored will again depend on the parameters of the SSC, but in any event would be more than a factor of ten beyond the capability of the Fermilab Tevatron.

The SSC will also be an exceedingly intense source of $b$ and $t$ quarks and thereby provide an opportunity for detailed and fundamental studies of their properties. The SSC may well be the first machine to produce $b$ quarks in sufficient quantities to permit a study of $\mathrm{CP}$ violation, a poorly understood phenomenon observed up to now only in kaon decay. If its mass is in excess of $150 \mathrm{GeV}$, the discovery of the $t$ quark may not be possible in presently available machines and will require a new generation of colliders such as the SSC. Discovery of additional, super-heavy quarks and leptons would also be possible only at a machine such as the SSC. 


\section{SSC PHYSICS REACH AS A FUNCTION OF ENERGY AND LUMINOSITY}

We evaluate in this section the impact of variations in energy and luminosity on the exploratory capability of the SSC. We do this by examining some representative examples of new physics. Such an analysis is by no means new. The review of Supercollider Physics by Eichten, Hinchliffe, Lane and Quigg, [3] the Conceptual Design Report, [1] the proceedings of the Snowmass [4] and Berkeley Detector Workshops [5] all provide a consistent picture of the physics potential of the SSC. The calculations presented here include an updated value for the lower bound on the $t$-quark mass, but the basic conclusions remain in substantial agreement with the earlier analyses. In particular, the energy dependence of various processes is well understood.

We present results as contours on a plot of SSC luminosity versus c.m. energy. Each contour represents a well-defined signal for a new particle of fixed mass or evidence for compositeness at a fixed energy scale. The signals correspond to a data run of $10^{7}$ seconds, obtainable during a typical calendar year of SSC operation. Two luminosity contours are also drawn in these plots. One represents the design value of $10^{33} \mathrm{~cm}^{-2} \mathrm{sec}^{-1}$ which is considered to be a reasonably conservative goal for the machine and detector technologies. This luminosity yields typically one or two proton-proton interactions per beam bunch crossing. The other contour represents an estimate of the maximum achievable luminosity as limited by beam-beam tune-shifts and synchrotron power dissipation. [6, 7] This maximum luminosity might be reached after several years of SSC operation. As discussed in the next section, such luminosities would place severe demands on 
presently available detector technologies. Since many events would be produced per bunch crossing, the difficulty of correctly assigning a set of detector signals to a particular process would increase in some cases. Nevertheless, the availability of luminosities above the design value may allow a significant extension of the exploratory reach of the SSC.

Our first example of new physics is a massive $Z^{\prime}$ boson, a carrier of a new force. We display in Figure 1 the luminosity required to produce $100 Z^{\prime}$ bosons per year as a function of the SSC c.m. energy. The contours are labelled with the mass of the $Z^{\prime}$. We have assumed Standard-Model couplings for the $Z^{\prime}$ so that 100 produced $Z^{\prime}$ particles would yield about six events in the cleanly observable decays to electron or muon pairs. As the SSC c.m. energy is varied from 30 to $40 \mathrm{TeV}$, the discovery reach in terms of the $Z^{\prime}$ mass increases from about 6.5 to 8.0 TeV at the design luminosity of $10^{33} \mathrm{~cm}^{-2} \mathrm{sec}^{-1}$. At a c.m. energy of $40 \mathrm{TeV}$, the mass reach could be increased to about $10 \mathrm{TeV}$ if the average luminosity is raised to $5 \times 10^{33} \mathrm{~cm}^{-2} \mathrm{sec}^{-1}$.

Figures 2 and 3 display similar plots for the Standard-Model Higgs boson. If the mass of the Higgs exceeds twice the mass of the $Z$ boson $\left(m_{Z}=91 \mathrm{GeV}\right)$, then its decay to $Z$ pairs will have a large branching ratio. These decays provide a very clean experimental signature consisting of four-lepton final states containing either electrons, muons, or both. Figure 2 shows contours corresponding to 20 such four-lepton events for Higgs masses of 400, 600 and $800 \mathrm{GeV}$. Above $800 \mathrm{GeV}$, the Higgs particle becomes a very broad effect and its discovery may require techniques more elaborate than searching for mass peaks. The Higgs production cross section was calculated assuming a $t$-quark mass of $85 \mathrm{GeV}$, a value 


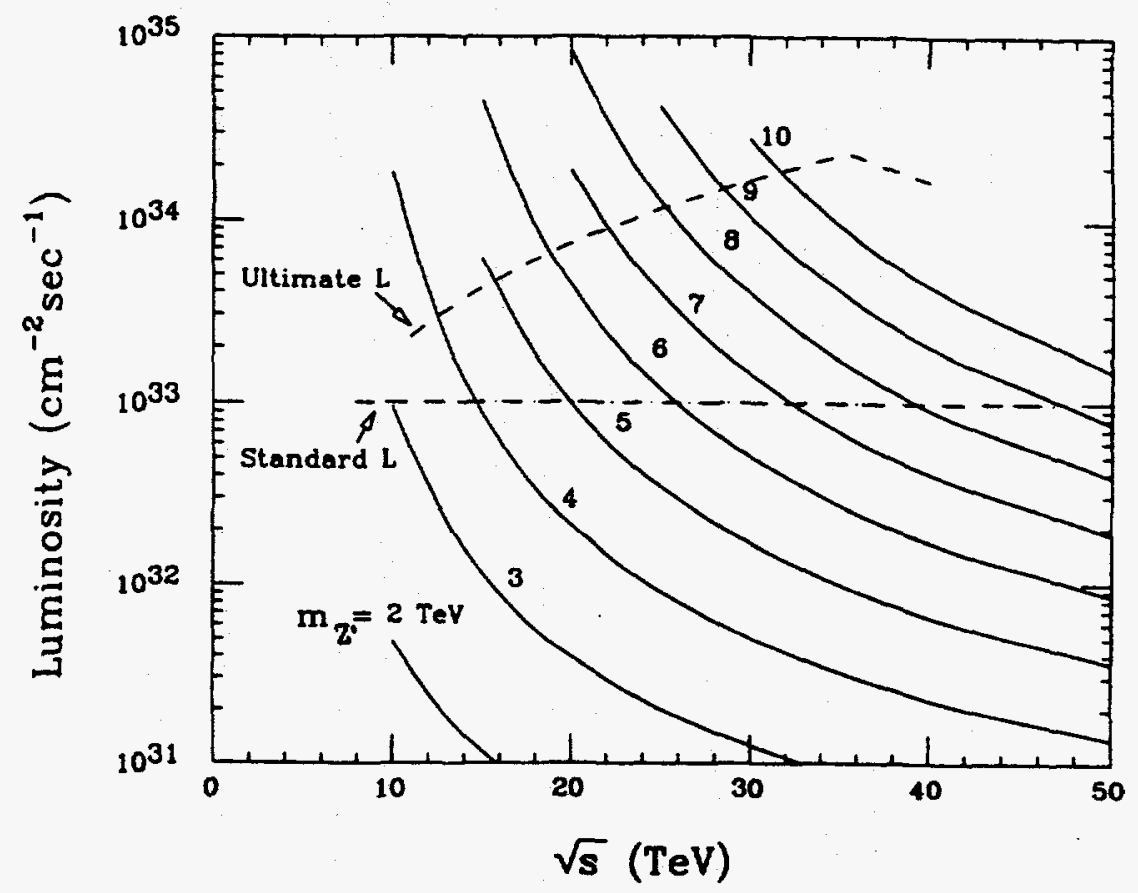

Figure 1. Luminosity required to produce in one year $100 Z^{\prime}$ bosons, with Standard Model couplings, as a function of c.m. energy. Results are shown for $Z^{\prime}$ masses in the range from 2 to $10 \mathrm{TeV}$.

somewhat above the present lower experimental limit. [8] If the $t$-quark mass is larger, the Higgs production cross section will increase, thereby extending the accessible Higgs mass range. To establish the existence of the Higgs convincingly will probably require somewhat more than 20 produced events because of $Z$-pair backgrounds and experimental inefficiencies. At the design luminosity, the Higgs mass reach of the $\mathrm{SSC}$ will therefore vary from about 600 to $800 \mathrm{GeV}$ as the c.m. energy increases from 30 to $40 \mathrm{TeV}$. The signal-to-background ratio improves by about $20 \%$ with energy over that same interval. Increases in luminosity above the design value will provide larger data samples that will allow more detailed studies of any observed signal. If the Higgs mass exceeds $1 \mathrm{TeV}$, the highest energy and luminosity will be required for its discovery. 


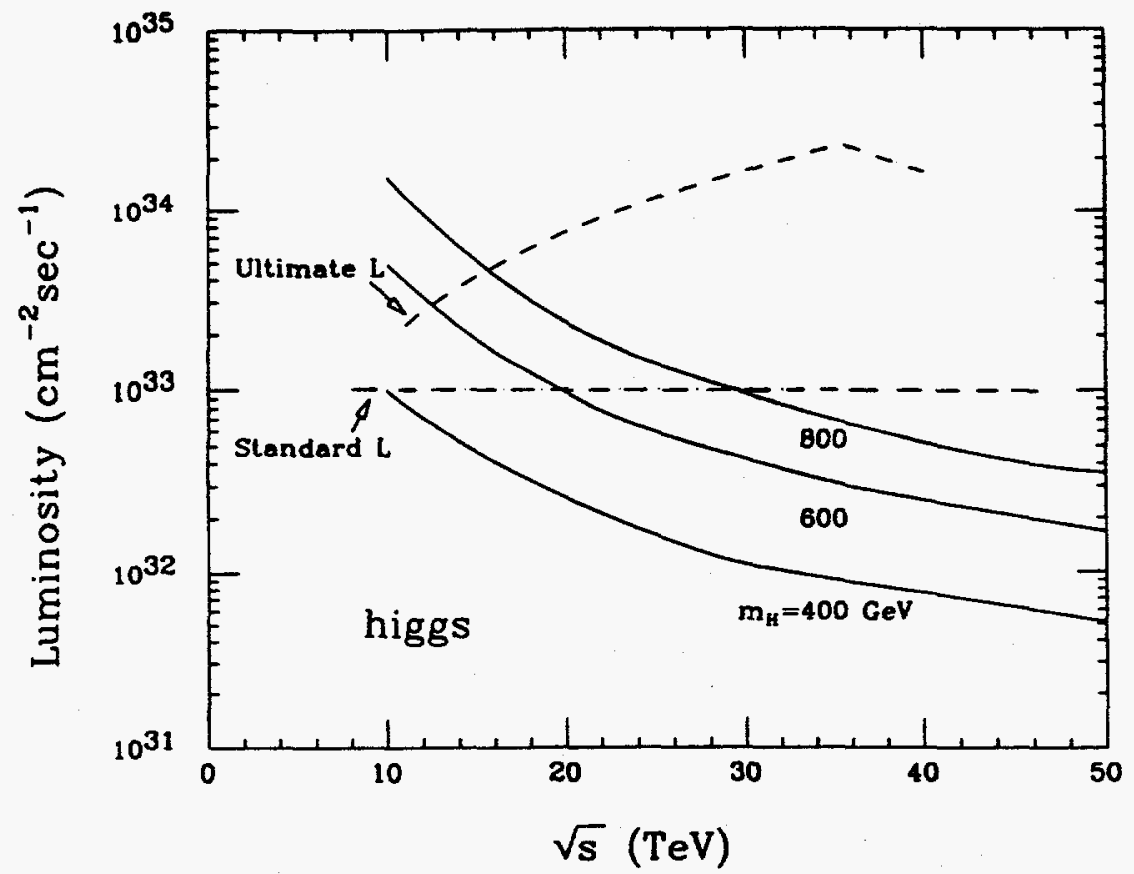

Figure 2. Luminosity required to produce in one year 20 Standard-Model Higgs bosons that decay to four charged leptons $(e, \mu)$ as a function of c.m. energy. (Mass of $t$ quark $=85 \mathrm{GeV}$.) Results are shown for Higgs masses of 400,600 and $800 \mathrm{GeV}$.

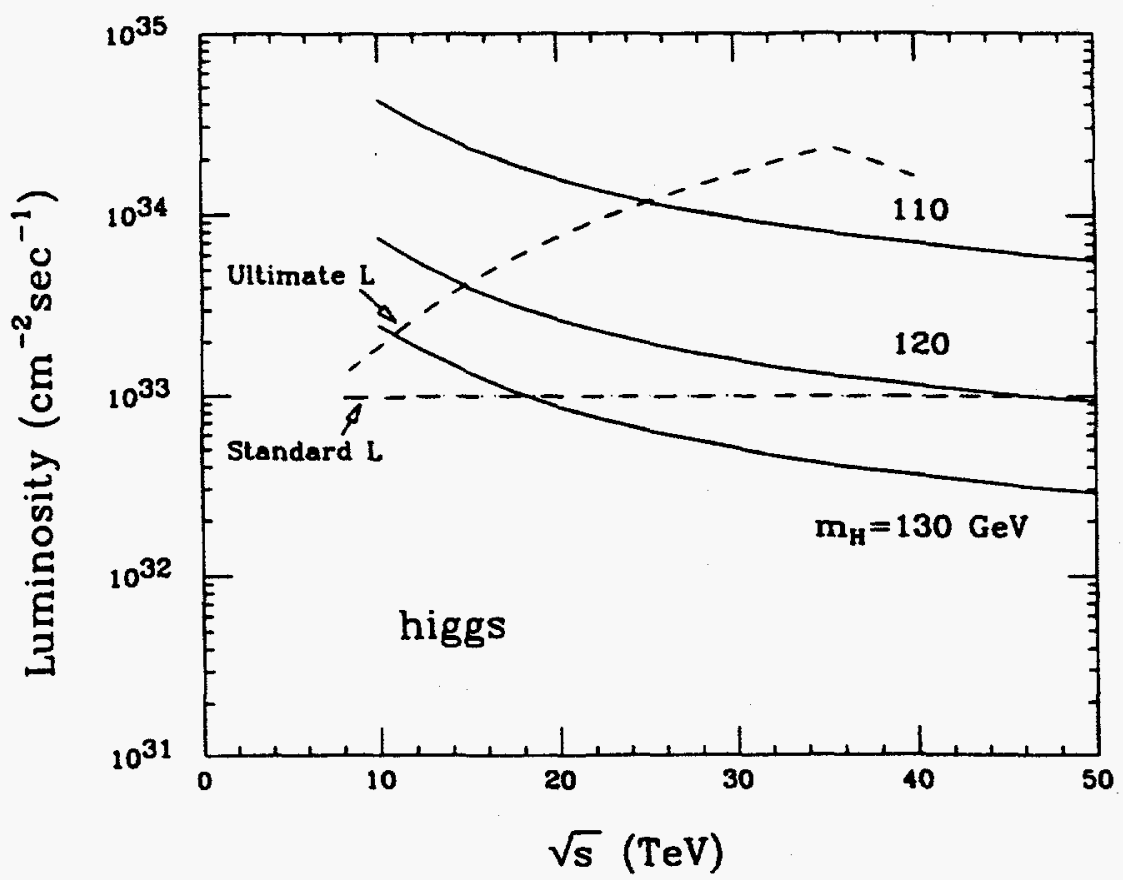

Figure 3. Luminosity required to produce in one year 40 intermediate mass Higgs bosons that decay into four charged leptons $(e, \mu)$ as a function of c.m. energy. (Mass of $t$ quark $=85 \mathrm{GeV}$.) Results are shown for Higgs masses of 110,120 and $130 \mathrm{GeV}$. 
If the Higgs mass is below $180 \mathrm{GeV}$, its detection becomes much more difficult because the decay to actual $Z$-boson pairs is no longer possible. The four-lepton decay mode becomes much rarer as the mass of the Higgs decreases. Figure 3 shows the plot for Higgs particles with low mass, where the contours represent 40 Higgs bosons which are produced and then decay to a four-lepton final state containing electrons, muons, or both. For this comparatively low-mass particle, the discovery reach in terms of mass depends only slightly on machine energy. Increases in luminosity can extend this reach, but may be difficult to utilize in this case because of the high rate of background events.

Supersymmetry is an elegant theory that postulates a relationship between the two basic classes of particles, fermions and bosons. Its discovery would revolutionize our conception of the fundamental space-time symmetry of the universe. The gluino is one example of a new supersymmetric particle. Because it is related to the gluon, it would be produced abundantly by strong interactions. However, its experimental signature is not as clean as those discussed above for the $Z^{\prime}$ and Higgs bosons, and a large number of events would be required to establish the existence of the gluino. Figure 4 shows the contour plot for the production of 10,000 gluino pairs for various masses. At the SSC design luminosity, the accessible gluino mass increases from about 1.35 to $1.60 \mathrm{TeV}$ as the c.m. energy is varied from 30 to $40 \mathrm{TeV}$. Present experimental limits on the gluino mass lie a factor of twenty below this value. [9]

A search for quark substructure or compositeness would consist of a measurement of hadronic jets at very large transverse momenta. A signal for compositeness would appear as an excess of events above the level predicted by the 


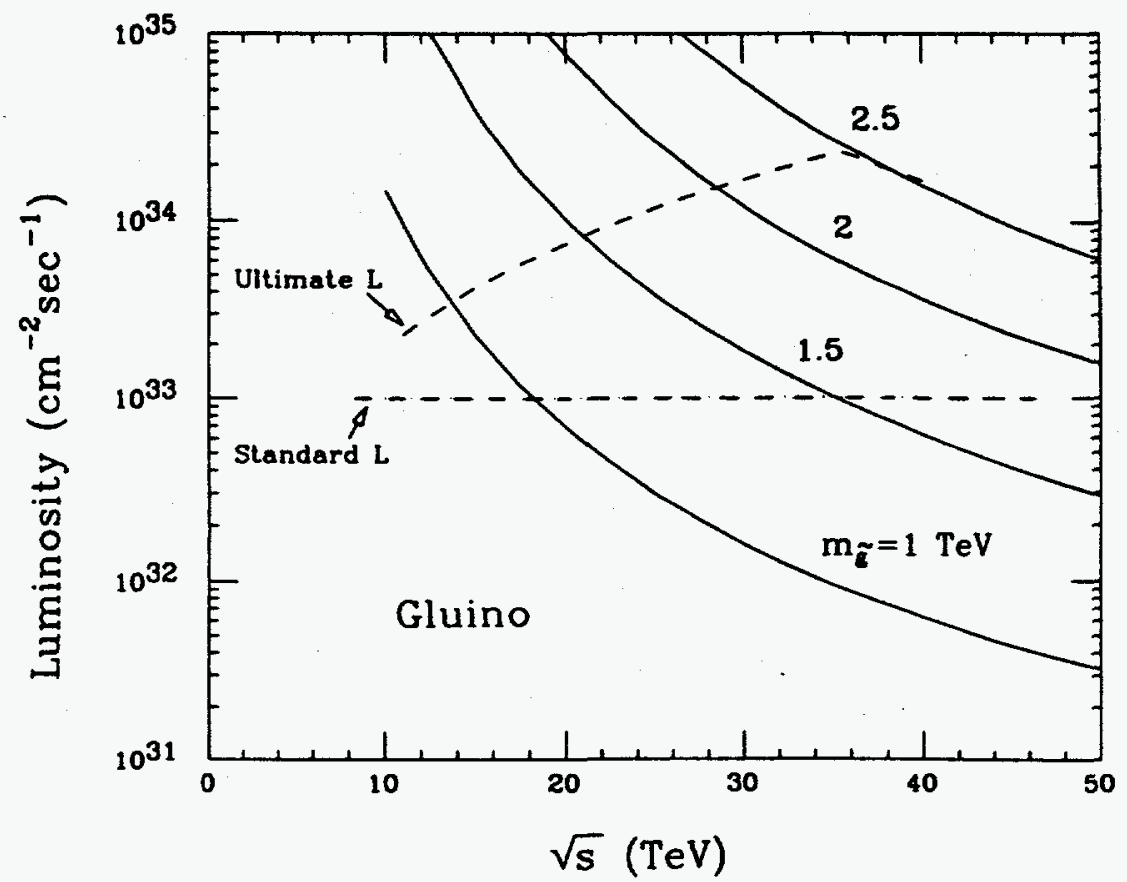

Figure 4. Luminosity required to produce 10,000 pairs of gluinos per year as a function of c.m. energy. Results are shown for gluinos masses in the range of 1.0 to $2.5 \mathrm{TeV}$.

theory of quark and gluon interactions known as QCD. As discussed above, the physical dimension at which substructure appears is parametrized by an energy scale, $\Lambda$, which is inversely proportional to the size of that substructure. Figure 5 shows the luminosity required to establish in one year compositeness scales of 10 , 20 and $30 \mathrm{TeV}$ as a function of c.m. energy. Here a signal of compositeness is defined to be more than 50 events in a transverse momentum interval of width $100 \mathrm{GeV}$ for which the observed signal is more than twice that expected from QCD alone. At the design luminosity, the sensitivity to compositeness changes from about 18 to $22 \mathrm{TeV}$ as the SSC c.m. energy varies from 30 to $40 \mathrm{TeV}$. The sensitivity at $40 \mathrm{TeV}$ can be raised to the $27 \mathrm{TeV}$ level by increasing the average luminosity to $5 \times 10^{33} \mathrm{~cm}^{-2} \mathrm{sec}^{-1}$. 


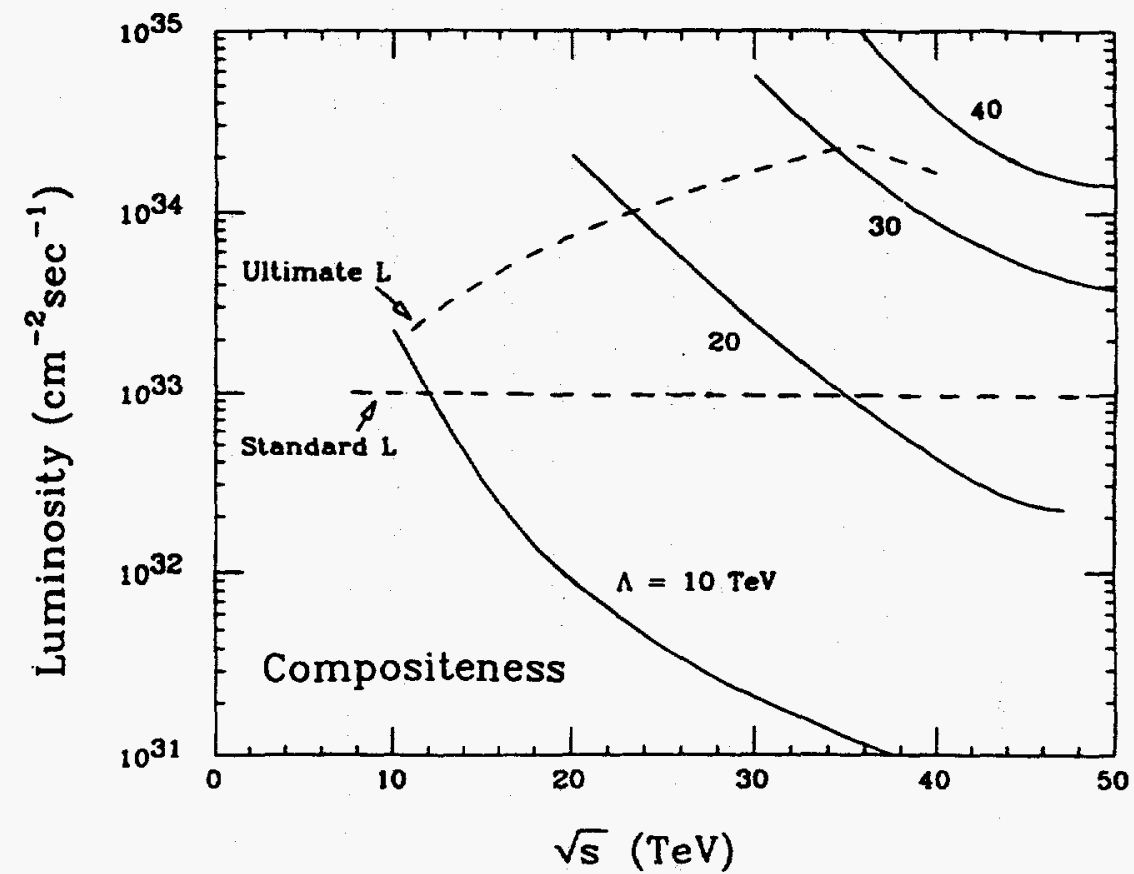

Figure 5. Luminosity required to establish in one year quark compositeness scales of $10,20,30$ and $40 \mathrm{TeV}$ as a function of c.m. energy.

As mentioned in the previous section, the SSC offers an opportunity for detailed studies of $b$ - and $t$-quark properties. The expected event rates are very large and do not depend strongly on the c.m. energy in the range from 30 to $40 \mathrm{TeV}$. At the design luminosity, approximately one trillion $b$ quarks will be produced per year and the ability of experiments to exploit this large rate is crucial if studies of $\mathrm{CP}$ violation are to be carried out.

The above examples of possible new physics are just a representative list for which reasonably accurate calculations can be made for the purpose of studying the impact of variations of the SSC energy and luminosity. It is important to remember that new physics may appear in a form that is not included in this inventory of examples. 


\section{EXPERIMENTAL IMPACT OF VARIATIONS IN SSC ENERGY AND LUMINOSITY}

The techniques for particle detection at SSC energies and luminosities have been discussed at numerous workshops. $[4,5]$ A number of detector configurations are now being investigated within the experimental community in anticipation of proposal preparations. The SSC places severe demands on the detectors because of the great event rates generated by the machine. At the design luminosity of $10^{33} \mathrm{~cm}^{-2} \mathrm{sec}^{-1}$ about 100 million events per second will be produced at each interaction region of which only a few are of interest in a typical particle search. The task of identifying these few events is just one of several experimental problems that must be solved. The great flux of secondary particles produced by the proton-proton interactions can obscure the signals of interest and can even cause significant radiation damage in the detector components. Techniques for overcoming such potential problems include the implementation of very fine detector segmentation, fast detector response times, and radiation-hard technologies.

In this report we are interested in the effect of variations in machine energy and luminosity on detector capabilities and design. Basically, the required detector performance is only weakly dependent on the SSC energy in the range from 30 to $40 \mathrm{TeV}$ but is significantly affected by the machine luminosity. It is therefore easier to accommodate changes in energy than increases in luminosity.

The energy and momentum scales associated with the detection of the decay fragments of a massive, new particle are set by the mass of such a particle and are essentially independent of machine energy when these fragments are detected 
at large angles. With increases in beam energy, it is of course possible to detect particles of greater mass and detector designs must take this into account. The length of the magnetic field region required to obtain a given fractional momentum resolution increases as the square root of the momentum. The required depth of calorimetry for shower containment or muon filtering increases only logarithmically with energy. For these reasons, no significant change in detector size is required for variations in the SSC c.m. energy in the range from 30 to $40 \mathrm{TeV}$.

Substantial increases in luminosity above the design value have a more severe impact on detector performance since the associated rate and radiation problems are directly proportional to the luminosity. Particularly vulnerable are tracking systems that directly surround the interaction region. Tracking devices based on proportional wire techniques represent a well-understood and proven technology and are expected to be viable at luminosities up to the SSC design value. At substantially higher luminosities, the occupancies and currents in such wire chambers reach a level that is likely to degrade their performance to unacceptable levels. It is conceivable, but not yet demonstrated, that large tracking systems based on silicon or scintillating fibers can be operated at these higher luminosities and be built for an acceptable cost. Experiments that depend critically on momentum measurements of charged particles within a few meters of the interaction point may not readily be able to take advantage of luminosity improvements beyond the design value.

Radiation damage is a major concern for calorimeters, devices that measure particle energies. This is particularly true for calorimeter elements located at small angles with respect to the SSC beams. Available calorimeter technologies 
differ in their radiation hardness. However, even robust designs such as those based on liquid argon must insure that their associated electronics can withstand the generated radiation doses. The question of radiation damage is presently under intense study and it is not yet possible to clearly define the operational limits for the various calorimeter technologies.

At a fixed distance from the interaction point, radiation levels rise steeply as the angle with respect to the beam direction is decreased. The efficient detection of massive, new particles requires full detector coverage to within about 5 degrees of the beam line. The expected radiation levels at 10 degrees are approximately five times lower than at 5 degrees. A five-fold increase in luminosity can therefore be accommodated with no additional radiation hardness if the detector coverage near the beams is reduced by about 5 degrees. In most cases, the resulting decrease in detection efficiency is more than offset by the increase in event rates.

The above discussion makes it clear that the impact of variations in luminosity above the design value are difficult to evaluate and will depend in a complicated way on the details of a particular detector design and technology choice. Muon detectors behind thick absorbers and calorimeters at angles greater than about 10 degrees with respect to the beam direction are expected to remain operational even at luminosities approaching the $10^{34} \mathrm{~cm}^{-2} \mathrm{sec}^{-1}$ level. If the calorimeter is designed to permit good electron identification and energy measurements, then some of the signals for new physics discussed in the previous section can be detected with such a combination of calorimetry and muon detection at luminosities substantially higher than the SSC design value. General-purpose detectors that incorporate radiation-hard calorimeters and external muon spectrometers can be 
expected to maintain a significant physics capability at such high luminosities even if certain elements, such as an interior wire-chamber tracking system, fail because of high particle rates.

\section{SUMMARY AND CONCLUSIONS}

We have examined the potential of the SSC to discover new physics as a function of both energy and luminosity. The opportunity for major discoveries is great as illustrated by several representative examples, including a new $Z$ boson, the Standard-Model Higgs particle, a supersymmetric particle (gluino), and quark compositeness. As the SSC c.m. energy is varied from 30 to $40 \mathrm{TeV}$, the relevant mass and compositeness scales that can be explored increase by about 25 to $30 \%$. Increasing the average luminosity by a factor of five above the design value of $10^{33} \mathrm{~cm}^{-2} \mathrm{sec}^{-1}$ at a given c.m. energy yields a similar increase of about $25 \%$ in the accessible mass and energy scales. Some particle searches may, however, not be feasible at such high luminosity. Typically event rates for producing very massive particles at fixed luminosity decrease by about $25 \%$ when reducing the $\mathrm{SSC}$ energy from 40 to $35 \mathrm{TeV}$. The corresponding rate decrease is approximately $50 \%$ when reducing the energy from 40 to $30 \mathrm{TeV}$.

It is clear that the ability to observe new physics depends on both the energy and luminosity of the SSC. The luminosity is, however, not an arbitrarily free parameter. Its peak value in the energy range from 30 to $40 \mathrm{TeV}$ is limited by machine physics considerations to a level of about $10^{34} \mathrm{~cm}^{-2} \mathrm{sec}^{-1}$. In this respect, the design value of $10^{33} \mathrm{~cm}^{-2} \mathrm{sec}^{-1}$ represents a reasonably conservative goal. The experimental techniques for the detection of proton-proton interactions 
at the SSC also impose limits on the peak luminosity at which the machine is operated. These limits depend on the details of the detector design and are therefore not easily quantified in a general way. As interaction rates increase, high detector occupancies, radiation damage, and multiple interactions must be confronted. It is fair to say that reasonable confidence exists within the experimental community that general-purpose detectors can be operated at luminosities up to the $10^{33} \mathrm{~cm}^{-2} \mathrm{sec}^{-1}$ level. More specialized detectors can probably cope with significantly higher luminosities.

The conclusions of the Committee are as follows: An SSC total energy anywhere in the range from 30 to $40 \mathrm{TeV}$ would provide an enormous increase in physics capability over existing machines. While the physics examples considered in this report do not show a dramatic dependence on energy in this range, it is clear that the discovery reach is enhanced significantly by operating the SSC at the highest possible energy. The luminosity that may ultimately be reached in this energy range is approximately one order of magnitude above the present design value of $10^{33} \mathrm{~cm}^{-2} \mathrm{sec}^{-1}$. Exploiting this capability will greatly extend the discovery potential of the SSC. We recommend that the planning of the SSC experiments take into account the possibility of such higher luminosities. It is important to remember that the SSC will be a major instrument of high energy physics during the early decades of the next century. Within our present knowledge of physics, there are no indications that would point toward a lower energy machine. Indeed, the lack of significant deviations from the Standard Model in existing data and our ignorance about electroweak symmetry breaking both ar- 
gue for exploring as large an energy range as possible. The design of the SSC should aim for the largest machine allowed by the available resources. 


\section{REFERENCES}

1. Superconducting Super Collider Conceptual Design, SSC-SR-2020 (1986)

2. D. Bintinger, Luminosity Limitations in Proceedings of the Workshop on Experiments, Detectors, and Experimental Areas for the Supercollider, Berkeley (1987)

3. E. Eichten, I. Hinchliffe, K. Lane and C. Quigg, Supercollider Physics, Rev. Mod. Phys. 56 (1984) 579

4. Proceedings of the 1984 Summer Study of the Design and Utilization of the Superconducting Super Collider, R. Donaldson and J.G. Morfin (eds.); Proceedings of the 1986 Summer Study on the Physics of the Superconducting Supercollider, R. Donaldson and J. Marx (eds.); Proceedings of the Summer Study on High Energy Physics in the 1990s, Snowmass (1988), S. Jensen (ed.)

5. Proceedings of the Workshop on Experiments, Detectors, and Experimental Areas for the Supercollider, Berkeley (1987), R. Donaldson and M.G.D. Gilchriese (eds.)

6. A. Chao, Possible Scenario to Reach Higher Luminosity, SSC-N-684 (1989)

7. R. Schwitters, Some Comments on SSC Ultimate Luminosity, (1989) 
8. F. Abe et al. (CDF Collaboration), to be published in Phys. Rev. Letters, give a lower limit of $77 \mathrm{GeV}$ on the t-quark mass.

9. F. Abe et al. (CDF Collaboration), Phys. Rev. Letters 62 (1989) 1825, give a lower limit of $73 \mathrm{GeV}$ on the gluino mass. 


\section{APPENDIX I \\ CHARGE TO AD HOC COMMITTEE ON SSC PHYSICS}

The Committee is asked to evaluate the impact on the SSC physics program of the following variation in machine parameters:

1) $30<E_{c m}<40 \mathrm{TeV}$ (in particular 30 and $35 \mathrm{TeV}$ compared to $40 \mathrm{TeV}$ )

2) $10^{33}<L_{\max }<5 \times 10^{33} \mathrm{~cm}^{-2} \mathrm{sec}^{-1}$

For the above range of c.m. energies, the Committee is asked to calculate expected cross sections for the following processes, taking into account the most recent lower limits on the $t$-quark mass:

a) Production of $t$ quarks

b) Higgs particle production

c) $Z$ pairs from standard reactions

d) Production of new, heavy gauge bosons

e) Jet production as a function of possible compositeness energy scales

f) Production of $b$-quarks with a view towards measuring CP violation

Whenever applicable and feasible, the Committee is asked to evaluate signalto-background ratios as a function of the SSC c.m. energy. A summary comparison of the "physics reach" at different energies and luminosities should be prepared. 
The Committee is also asked to examine the viability of detector techniques at luminosities in the above range. In particular, what is the impact on:
a) Tracking
b) Calorimetry
c) Muon detection
d) Data acquisition

as the luminosity is increased above the design value of $10^{33} \mathrm{~cm}^{-2} \mathrm{sec}^{-1}$.

A draft of a written report is requested by the end of the meeting on December 1, 1989. 


\section{APPENDIX II}

\section{MEMBERSHIP OF AD HOC COMMITTEE ON SSC PHYSICS}
1) R. Cahn
LBL
2) D. Cassel
Cornell University
3) A. Chao
SSC
4) M.G.D. Gilchriese
$\mathrm{SSC}$
5) F. Gilman
SLAC
6) H. Gordon
BNL
7) J. Gunion
UC Davis
8) I. Hinchliffe
LBL
9) R. Hollebeek
University of Pennsylvania
10) G. Kane
University of Michigan
11) M. Marx
SUNY Stony Brook
12) L. Nodulman
SSC/ANL
13) R. Thun
SSC/Michigan 